\title{
MicroRNA discovery by similarity search to a database of RNA-seq profiles
}

\author{
Sachin Pundhir and Jan Gorodkin* \\ Center for non-coding RNA in Technology and Health, Department of Veterinary Clinical and Animal Sciences (IKVH), University of Copenhagen, \\ Frederiksberg C, Denmark
}

\section{Edited by:}

Panayiota Poirazi, Foundation for Research and Technology-Hellas, Greece

Reviewed by:

Martin Reczko, Synaptic Ltd. Greece

Pavlos Pavlidis, Foundation for Research and Technology-Hellas, Greece

*Correspondence:

Jan Gorodkin, Department of Veterinary Clinical and Animal Sciences (IKVH), Center for non-coding RNA in Technology and Health, IKVH, University of Copenhagen, Gronnegårdsvej 3 , 1870 Frederiksberg C, Denmark e-mail:gorodkin@rth.dk
In silico generated search for microRNAs (miRNAs) has been driven by methods compiling structural features of the miRNA precursor hairpin, as well as to some degree combining this with the analysis of RNA-seq profiles for which the miRNA typically leave the drosha/dicer fingerprint of 1-2 22 nt blocks of reads corresponding to the mature and star miRNA. In complement to the previous methods, we present a study where we systematically exploit these patterns of read profiles. We created two datasets comprised of 2540 and 4795 read profiles obtained after preprocessing short RNA-seq data from miRBase and ENCODE, respectively. Out of 4795 ENCODE read profiles, 1361 are annotated as non-coding RNAs (ncRNAs) and of which 285 are further annotated as miRNAs. Using deepBlockAlign (dba), we align ncRNA read profiles from ENCODE against the miRBase read profiles (cleaned for "self-matches") and are able to separate ENCODE miRNAs from the other ncRNAs by a Matthews Correlation Coefficient (MCC) of 0.8 and obtain an area under the curve of 0.93 . Based on the dba score cut-off of 0.7 at which we observed the maximum MCC of 0.8 , we predict 523 novel miRNA candidates. An additional RNA secondary structure analysis reveal that 42 of the candidates overlap with predicted conserved secondary structure. Further analysis reveal that the 523 miRNA candidates are located in genomic regions with MAF block (UCSC) fragmentation and poor sequence conservation, which in part might explain why they have been overlooked in previous efforts. We further analyzed known human and mouse miRNA read profiles and found two distinct classes; the first containing two blocks and the second containing $>2$ blocks of reads. Also the latter class holds read profiles that have less well defined arrangement of reads in comparison to the former class. On comparison of miRNA read profiles from plants and animals, we observed kingdom specific read profiles that are distinct in terms of both length and distribution of reads within the read profiles to each other. All the data, as well as a server to search miRBase read profiles by uploading a BED file, is available at http://rth.dk/resources/mirdba.

Keywords: microRNA, miRNA read profiles, RNA-seq, alignment, deepBlockAlign, read profiles

\section{INTRODUCTION}

MicroRNAs (miRNAs) are small, non-coding RNAs 18-24 nucleotides in length that play important roles in various biological and metabolic processes, including signal transduction, developmental timing, cell maintenance and differentiation (Zhang et al., 2006b). MiRNAs are involved in post-transcriptional regulation of gene expression by directly cleaving targeted mRNAs or repressing translation (Bartel, 2004). Many in-vitro and insilico based approaches have been developed for the prediction of miRNAs. In-vitro based approaches like genetic screening approach have contributed to many founding members of miRNAs. However, due to low efficiency and high cost, these are limited for wider applications. Many in-silico based approaches have been developed based on major characteristic of miRNAs for example hairpin-shaped stem loop structure integrated with homology search (Wang et al., 2005; Dezulian et al., 2006) or evolutionary conservation (Lai et al., 2003; Lim et al., 2003). Besides, methods based on phylogenetic shadowing (Berezikov et al., 2005), neighbor step loop search (Ohler et al., 2004), minimal folding free energy index (Zhang et al., 2006a) and machine learning approaches have also been developed (Table 1). Various plant and animal miRNAs have been identified using these computational approaches. However, many of these methods have sensitivity problems and give a number of false positive results (Bentwich, 2005). Taken together all search methods aim to reduce the search space in their own respective ways (Lindow and Gorodkin, 2007).

Recent advances in high throughput sequencing have provided a new opportunity for genome annotation including prediction of novel miRNAs. Many tools like miRDeep2 (Friedländer et al., 2012), miRDeep* (An et al., 2013), and miRanalyzer (Hackenberg et al., 2011) exploit the aggregated set of RNAseq reads along with secondary structure potential to annotate a genomic locus as miRNA. Indeed, these tools have great ability to predict novel miRNA genes (Williamson et al., 2013). However, these tools do not completely exploit the subtle differences in 
Table 1 | Major approaches for the computational prediction of micro-RNA.

\begin{tabular}{|c|c|c|}
\hline Approach & Programs & References \\
\hline Evolutionary conservation and stem loop structure & miRseeker and miRscan & Lai et al., 2003; Lim et al., 2003 \\
\hline Neighbor stem loop search & - & Ohler et al., 2004 \\
\hline Sequence based homology and stem loop structure & microHARVESTER, MiRAlign & Wang et al., 2005; Dezulian et al., 2006 \\
\hline Phylogenetic shadowing & - & Berezikov et al., 2005 \\
\hline Minimum free energy index & - & Zhang et al., 2006a \\
\hline Machine learning methods & ProMiR, mirCoS-a, MiPred & Nam et al., 2005; Jiang et al., 2007; Sheng et al., 2007 \\
\hline RNA-seq based & miRanalyzer, miRDeep2, miRDeep* & $\begin{array}{l}\text { Hackenberg et al., 2011; Friedländer et al., 2012; An et al., } \\
2013\end{array}$ \\
\hline
\end{tabular}

the arrangement of reads mapped to pre-miRNA. Furthermore, many of these tools start by identifying potential precursor locus for miRNA based on strict rules like fixed precursor size of $110 \mathrm{bp}$ or loop region of size $15 \mathrm{bp}$. This may result in unconventional miRNA patterns like miRNA-offset RNAs (moRs) that encode for up to four distinct, stable small RNAs (Shi et al., 2009) or plant miRNAs that have different biogenesis mechanism be readily missed by these tools (Lelandais-Briere et al., 2010). Furthermore, many microRNA-sized small RNAs have also been reported to be commonly produced not only from miRNA precursors but also from most other classes of structured RNAs like snoRNA and tRNA (Kawaji et al., 2008; Taft et al., 2009).

Several recent studies have recognized that short RNA-seq data, when mapped back to the host genome form read coverage patterns that are distinct and can be used to distinguish between major non-coding RNAs (ncRNAs) such as miRNA, snoRNA and tRNA (Erhard and Zimmer, 2010; Jung et al., 2010; Langenberger et al., 2010, 2012). These read coverage patterns have been referred to as "read profile" or block group (see also Figure 2) and are composed of distinctive clusters of reads (blocks) with similar start and/or stop position. The read profiles are often influenced by chemical modifications like in the case of tRNAs (Findeiß et al., 2011), or by secondary structures like in the case of miRNAs where miR and $\mathrm{miR}^{*}$ products mutually position with a $3^{\prime}$-overhang that is characteristic for dicer cleavage (Figure 1).

In this study, we present a novel strategy based on pairwise alignment of two read profiles, deepBlockAlign (Langenberger et al., 2012) to predict putative miRNAs in the human genome. We apply this approach on an extensive dataset of read profiles derived from 18 short RNA-seq experiments from ENCODE (ENCODE Consortium, 2011, 2012), and present some putative miRNAs that showed significant similarity to read profiles of known miRNAs from miRBase. We also show distinct classes of miRNA read profiles identified through alignment and hierarchical clustering of read profiles from human and mouse. Furthermore, we present miRNA read profiles that are specific to animals and plants.

\section{MATERIALS AND METHODS \\ 2.1. DATASETS}

We downloaded two RNA-seq datasets for the analysis of read profiles. First dataset is comprised of short-reads mapped to

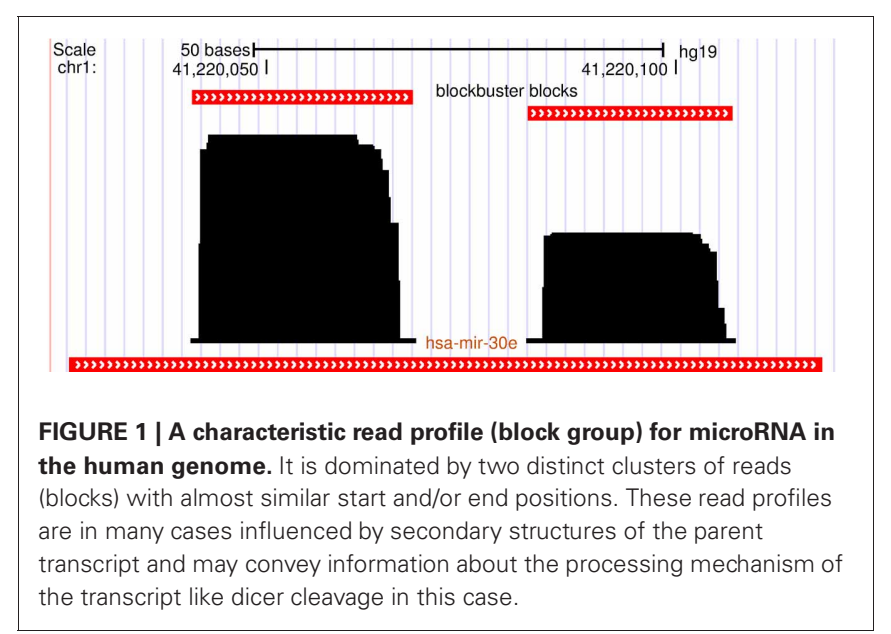

the human (hg19, Feb. 2009) genome assembly and is downloaded in BAM format from the ENCODE database (ENCODE Consortium, 2011, 2012). This dataset is comprised of 18 RNAseq experiments performed on nine human tissues with each having two biological replicates. It is to be noted that prior to sequencing, these biological replicates have been grown and isolated independently. In the following, we will refer to this dataset as ENCODE dataset (Table 2). Second dataset is comprised of short-reads mapped to 4862 distinct miRNAs from 20 organisms in miRBase (Kozomara and Griffiths-Jones, 2011). The short reads are derived from 244 GEO experiments performed on various tissues. In the following, we will refer to this dataset as miRBase dataset (Table 3).

\subsection{PREPROCESSING OF DATASET}

Both datasets were subjected to two pre-processing steps. Firstly, the mapped reads were formatted into BED format. The formatting was done for each of the 18 experiments from ENCODE and 20 organisms from the miRBase dataset, separately (Figures 2A,B). Secondly, each BED format file was processed to identify distinct accumulation of reads by assigning two reads to the same locus, when they were separated by less than $40 \mathrm{nt}$. We chose $40 \mathrm{nt}$ as the threshold to consider two reads from separate loci based on two observations (a) most short reads are less than $40 \mathrm{nt}$ in length and two genomic loci separated by a region of $>40 \mathrm{nt}$ with no mapped reads can most likely be considered as distinct. (b) the loop region of most pre-miRNAs 
Table 2 | The ENCODE dataset is comprised of short-reads from 18 RNA-seq experiments performed on nine human tissues with each having two replicates.

\begin{tabular}{|c|c|c|c|c|}
\hline \multirow[t]{2}{*}{$\mathrm{Tis}^{\mathrm{a}}$} & \multicolumn{2}{|c|}{ Replicate1 } & \multicolumn{2}{|c|}{ Replicate2 } \\
\hline & \# reads ${ }^{b}$ & $\# B G s^{c}$ & $\#$ reads ${ }^{b}$ & $\# B G s^{c}$ \\
\hline $\mathrm{Bl}$ & $49,280,641$ & 16,437 & $56,439,584$ & 19,609 \\
\hline $\mathrm{Br}$ & $48,773,897$ & 15,148 & $48,394,385$ & 13,317 \\
\hline $\mathrm{Bt}$ & $26,713,326$ & 13,309 & $40,144,816$ & 13,555 \\
\hline$C x$ & $41,301,918$ & 15,890 & $40,798,294$ & 15,948 \\
\hline Ep & $47,775,551$ & 13,522 & $44,163,861$ & 10,923 \\
\hline Es & $35,965,377$ & 12,692 & $33,651,242$ & 14,697 \\
\hline Li & $31,930,869$ & 6158 & $33,939,724$ & 10,684 \\
\hline Lu & $38,877,787$ & 14,511 & $43,732,746$ & 15,873 \\
\hline Sn & $37,649,014$ & 6370 & $41,022,882$ & 11,268 \\
\hline \multirow{2}{*}{\multicolumn{5}{|c|}{$\begin{array}{l}\text { The dataset was downloaded from ENCODE (ENCODE Consortium, 2011) and } \\
\text { is composed of a uniform read length of } 36 \mathrm{nt} \text {. }\end{array}$}} \\
\hline & & & & \\
\hline \multicolumn{5}{|c|}{ a Tissues with each having two biological replicates (Bl, Blood; Br, Brain; Bt, } \\
\hline \multicolumn{5}{|c|}{ Breast; Cx, Cervix; Ep, Epithelium; Es, Embryonic stem cell; Li, Liver; Lu, Lung; } \\
\hline \multicolumn{5}{|c|}{ Sn, Skin). } \\
\hline \multicolumn{5}{|c|}{${ }^{b}$ Number of mapped reads. } \\
\hline
\end{tabular}

is $<40 \mathrm{nt}$ in length. Consecutive reads within a locus were divided into blocks using blockbuster (with parameters: distance 40,-minBlockHeight 2, -minClusterHeight 10, -scale 0.5) (Langenberger et al., 2009) (Figure 2C). blockbuster merges mapped reads into blocks based on their location in the reference genome. Thus, stacks of reads are combined to read blocks which is analogous to tags (set of reads) processed from a specific locus. This strategy greatly reduces the size of the data set and allows the application of more costly algorithms while maintaining structural properties such as position, length and approximate read start sites and ends. The obtained set of one or more blocks at a locus are then called block groups (Table 2). It is to be noted that for the ENCODE dataset, we discarded all blocks that had read count of $<10 \%$ with respect to the total reads within its block group in order to (a) ensure all blocks are represented by at least one read, since minimum number of reads in a block group is set to 10 (-minClusterHeight 10); and, (b) nullify the effect of sequencing depth during comparison of block groups across 18 RNA-seq experiments from the ENCODE dataset (Figure 2A). Here after, we will use the term block group and read profile, synonymously.

Of the 3838 block groups obtained after the preprocessing of miRBase dataset, we filtered 2540 block groups that had more than one block and were $\leq 200 \mathrm{nt}$ in length. The 2540 block groups were then compiled to from a database of miRNA read profiles (Figure 2B and Table 3) abbreviated hereafter as miRRPdb. Next, for the ENCODE dataset, we derived 58,161 genomic loci where a block group or read profile is observed in at least one tissue (Figure 2A). For each genomic locus, we retrieved one block group corresponding to the tissue in which the block group had the maximum number of blocks leaving us with 58,161 block groups. All the block groups were then
Table 3 | miRBase dataset is comprised of short-reads mapped to 4862 distinct microRNAs from 20 organisms.

\begin{tabular}{|c|c|c|c|c|}
\hline \multirow[t]{2}{*}{ Org $^{a}$} & \multirow[t]{2}{*}{ \# reads ${ }^{b}$} & \multirow[t]{2}{*}{$\# \mathrm{miR}^{\mathrm{c}}$} & \multicolumn{2}{|c|}{ \# block groups } \\
\hline & & & All & Filter ${ }^{\mathrm{e}}$ \\
\hline Ame & $475,288(1)$ & 159 & 109 & 96 \\
\hline Ath & 472,5021 (8) & 275 & 303 & 173 \\
\hline $\mathrm{Bfl}$ & $37,217(1)$ & 113 & 71 & 34 \\
\hline Bmo & $2,021,309(3)$ & 384 & 264 & 194 \\
\hline $\mathrm{Cbr}$ & $17,442(1)$ & 115 & 81 & 25 \\
\hline $\mathrm{Cel}$ & $1,048,509(6)$ & 184 & 130 & 93 \\
\hline $\mathrm{Cqu}$ & $379,978(1)$ & 68 & 61 & 29 \\
\hline Cre & $1082(1)$ & 28 & 19 & 11 \\
\hline $\mathrm{Crm}$ & $9988(1)$ & 95 & 63 & 19 \\
\hline Cte & $50,659(1)$ & 118 & 72 & 8 \\
\hline Dme & $35,664,132(50)$ & 237 & 48 & 45 \\
\hline Hsa & $81,138,802(79)$ & 1279 & 801 & 550 \\
\hline Mmu & $913,716,590(82)$ & 749 & 688 & 624 \\
\hline Nve & $2711(1)$ & 34 & 17 & 2 \\
\hline Osa & $1,506,288(4)$ & 440 & 540 & 288 \\
\hline $\mathrm{Ppc}$ & $11,176(1)$ & 113 & 66 & 5 \\
\hline Ppt & $503,573(3)$ & 4224 & 285 & 148 \\
\hline Rco & $147(25)$ & 13 & 2 & 0 \\
\hline Spu & $6458(1)$ & 38 & 25 & 7 \\
\hline Tca & $4,861,929(2)$ & 196 & 193 & 189 \\
\hline Total & $1,046,178,299$ & 4862 & 3838 & 2540 \\
\hline
\end{tabular}

Dataset was downloaded from miRBase (Kozomara and Griffiths-Jones, 2011). ${ }^{a}$ Ame, A. mellifera; Ath, A. thaliana; Bfl, B. floridae; Bmo, B. mori; Cbr, C. briggsae; Cel, C. elegans; Cqu, C. quinquefasciatus; Cre, C. reinhardtii; Crm, C. remanei; Cte, C. teleta; Dme, D. melanogaster; Hsa, H. sapiens; Mmu, M. musculus; Nve, N. vectensis; Osa, O. sativa; Ppc, P. pacificus; Ppt, P. patens; Rco, R. communis; Spu, S. purpuratus; Tca, T. castaneum.

${ }^{b}$ Number of mapped reads. Total number of GEO experiments are given in brackets. Some experiments are comprised of reads from multiple organisms.

${ }^{c}$ Total number of distinct miRNAs with mapped reads.

dTotal number of block groups or miRNAs retrieved after preprocessing.

eBlock groups with $>1$ block and $\leq 200 \mathrm{nt}$ in length that are compiled to form a database of miRNA read profiles (miRRPdb).

compared to known annotation [1049 miRNA from miRBase v16 (Kozomara and Griffiths-Jones, 2011), 513 tRNA loci from gtRNAdb (Chan and Lowe, 2009), 402 snoRNA, 1794 scRNA, 2007 snRNA loci and 722 other RNAs from UCSC annotation (Karolchik et al., 2004)]. Block groups were also compared with 8811 ncRNA annotations from Rfam (Gardner et al., 2011). All the block groups whose coordinate overlapped at $\geq 1 \mathrm{nt}$ with that of known annotations were designated as "annotated" block groups (Table 4). Similarly, block groups were compared with coordinates of exon, intron, $5^{\prime}$ UTR and $3^{\prime}$ UTR region downloaded from UCSC (Karolchik et al., 2004) and were annotated accordingly, if overlapping at $>50 \%$ else designated as from intergenic region. If a block group overlaps to more than two genomic regions, then the region with maximum overlap is assigned to it. Of the 58,161 block groups, we filtered 4795 block groups that had more than one block and were $\leq 200 \mathrm{nt}$ in length. Out of 4795 


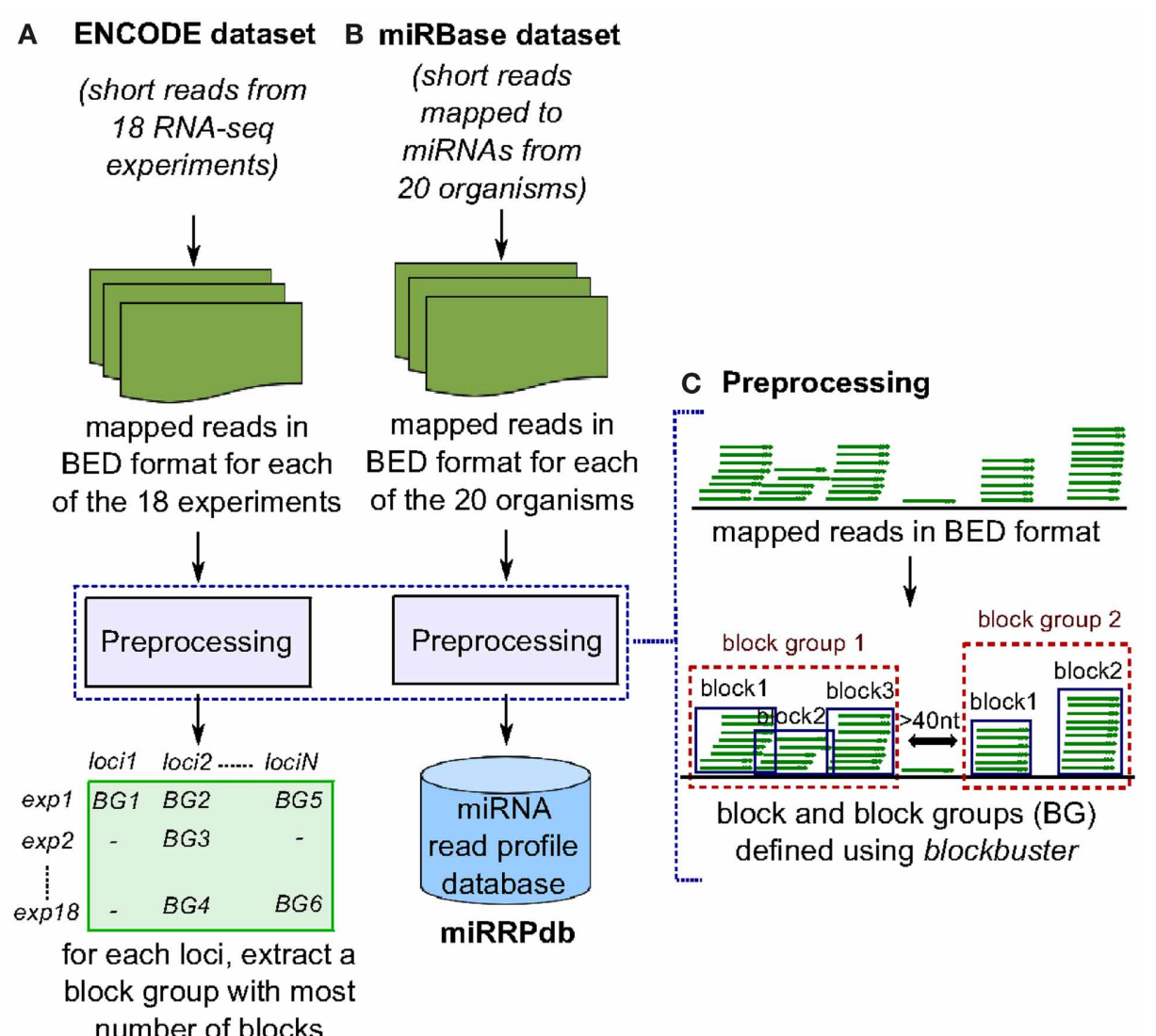

FIGURE 2 | Preprocessing of ENCODE and miRBase datasets. (A) In ENCODE dataset, reads mapped to human genome from each of the 18 RNA-seq experiments were subjected to preprocessing to obtain closely spaced set of reads termed here as "block group". Block groups thus obtained were compiled so as to identify a set of distinct genomic loci that have a block group in at least one experiment. Next, for each locus, we retrieved one block group corresponding to the experiment in which the block group has maximum number of blocks leaving us with 58,161 block groups. (B) In miRBase dataset, reads mapped to microRNAs from each of the 20 organisms were subjected to preprocessing and block groups thus obtained were compiled as miRNA read profile database (miRRPdb). (C) Given a set of mapped reads in BED format, we derive closely spaced stack of reads termed here as "block group" using blockbuster (Langenberger et al., 2009). Each block group or read profile is composed of one or more blocks of reads.
Table 4 | Annotation status of 58,161 block groups obtained after preprocessing of ENCODE dataset and their alignment to miRRPdb.

\begin{tabular}{llll}
\hline Annotation & \multicolumn{2}{c}{ \# block groups } & \# miRRPdb hits(\%) \\
\cline { 2 - 3 } & Alla & Filter $^{\mathbf{b}}$ & \\
\hline miRNA & 571 & 285 & $223(78)$ \\
snoRNA & 468 & 255 & $3(1)$ \\
tRNA & 625 & 496 & $7(1)$ \\
snRNA & 395 & 143 & $6(4)$ \\
scRNA & 187 & 46 & $3(7)$ \\
others & 277 & 136 & $8(6)$ \\
unannotated & 55,638 & 3434 & $523(15)$ \\
Total & 58,161 & 4795 & $773(16)$ \\
\hline
\end{tabular}

a Block groups obtained after preprocessing and are overlapping to non-coding RNA annotation.

${ }^{b}$ Block groups with $>1$ block and $\leq 200 \mathrm{nt}$ in length.

${ }^{c}$ Block groups that have significant alignment score $(\geq 0.7)$ to miRNA read profile database (miRRPdb). block groups, 1361 were annotated, and the rest 3434 were unannotated, see Table 4. We used the 1361 annotated block groups as benchmark dataset to evaluate the prediction performance of our method.

\subsection{PERFORMANCE EVALUATION}

The performance of the proposed method for the prediction of miRNA is evaluated based on sensitivity, specificity and MCC that are computed using a confusion matrix $(2 \times 2$ contingency table). The confusion matrix is essentially composed of four components (a) number of miRNA that are correctly predicted as miRNA (True Positive, TP), (b) number of miRNA that are incorrectly predicted as non-miRNA (False Negative, $\mathrm{FN}$ ), (c) number of non-miRNA that are incorrectly predicted as miRNA (False Positive, FP); and, (d) number of non-miRNA that are correctly predicted as non-miRNA (True Negatives, $\mathrm{TN})$. The sensitivity measures the proportion of true positives (TP) out of total number of positives (miRNAs) in the benchmark dataset $(\mathrm{TP} / \mathrm{TP}+\mathrm{FN})$. Like-wise, specificity measures the 
proportion of true negatives (TN) out of total number of negatives (non-miRNAs) in the benchmark dataset [TN/(TN + FP)]. The MCC is a discrete version of Pearson's correlation coefficient and is widely used in machine learning to measure the quality of (two-class) binary classifications. It is computed as

$$
\mathrm{MCC}=\frac{(\mathrm{TP} \times \mathrm{TN})-(\mathrm{FP} \times \mathrm{FN})}{\sqrt{(\mathrm{TP}+\mathrm{FP})(\mathrm{TP}+\mathrm{FN})(\mathrm{TN}+\mathrm{FP})(\mathrm{TN}+\mathrm{FN})}}
$$

\subsection{PREDICTION OF PUTATIVE miRNA}

In an earlier study, we have developed a tool named deepBlockAlign for the alignment of two read profiles (Langenberger et al., 2012). deepBlockAlign normalizes the read counts by the total reads within a block group followed by a two-tier strategy to align read profiles. The alignment score from deepBlockAlign ranges from 0 , suggesting perfect dissimilarity, to 1 for perfect similarity between the two read profiles. It is to be noted that in the absence of any statistical power or background distribution to evaluate the significance of deepBlockAlign scores (since "background" transcription still has to be defined) (Langenberger et al., 2012), we have used deepBlockAlign to compare the 1361 annotated read profiles from ENCODE dataset against miRRPdb in order to identify a meaningful discriminative dba score (see section 3.1 below). Based on the derived dba score, we align 3434 unannotated read profiles from ENCODE dataset against miRRPdb and identify novel genomic regions that have read profiles similar to those of known miRNAs (Figure 3A).

\subsection{PREDICTION OF miRNA FAMILIES}

To predict distinct classes of miRNA based on their read profiles, we performed cluster analysis on 550 human and 624 mouse read profiles from miRRPdb, separately. In cluster analysis, we first perform all vs. all alignment of all block groups using deepBlockAlign to generate a square matrix of alignment scores. Second, the R package pvclust (Suzuki and Shimodaira, 2006) is used for hierarchical clustering of block groups based on their alignment scores (Figure 3B). pvclust computes the $p$ value for each cluster in hierarchical clustering using multiscale bootstrap resampling and indicates how strong the cluster is supported by the data. We select all the clusters comprised of at least 15 read profiles at a $p$-value of $<0.05$, as families of miRNAs that share similar read profiles. To predict any organism specific read profile class, we also performed the cluster analysis on all 2540 read profiles from miRRPdb.

\section{RESULTS}

\subsection{BENCHMARKING}

To benchmark the prediction performance of our proposed method for the prediction of putative miRNAs, we aligned 1361 annotated read profiles (285 miRNA and 1076 other ncRNAs; Table 4) from ENCODE dataset against miRRPdb using

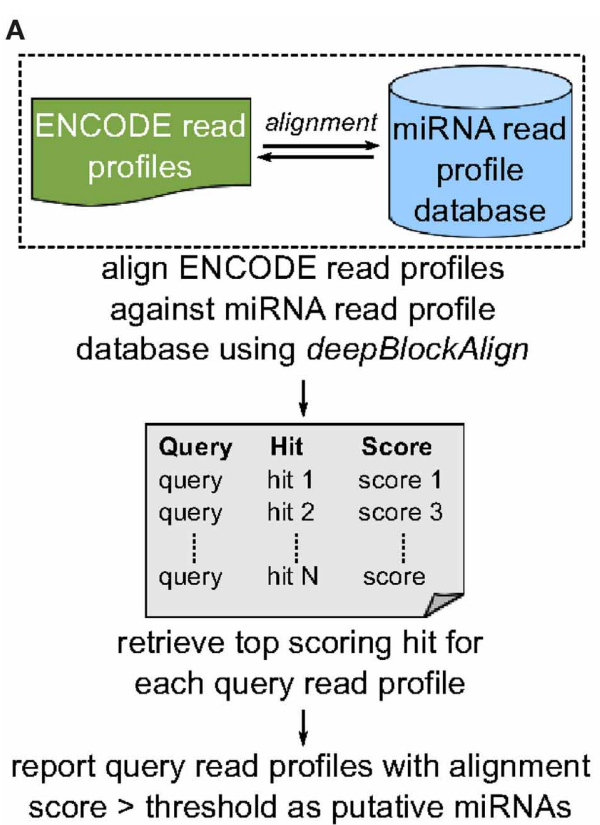

FIGURE 3 | Search strategy. (A) To predict putative miRNA candidates, we align 4795 read profiles from ENCODE dataset against miRNA read profile database (miRRPdb) that comprise of 2540 block groups using deepBlockAlign. We retrieve top scoring alignment for each query as putative miRNA, if the score is above the threshold of 0.7. (B) To predict distinct classes of miRNA based on their read profiles, all 2540 read profiles from miRRPdb were aligned against each other using

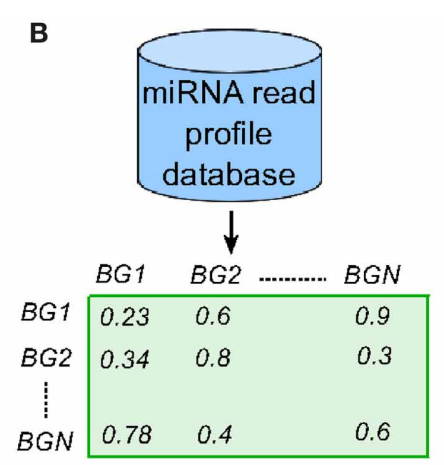

all vs all alignment of block groups using deepBlockAlign

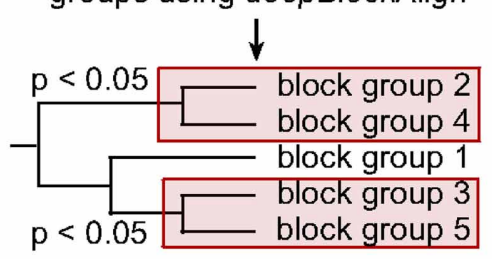

hierarchical clustering using pvclust
deepBlockAlign to generate an alignment score matrix. Next, we perform hierarchical clustering of block groups based on their alignment score using pvclust. pvclust computes the p-value for each cluster in hierarchical clustering using multiscale bootstrap resampling and indicates how strong the cluster is supported by the data. We select all the clusters with $p<0.05$ that represent families of microRNAs that share similar read profiles. 
deepBlockAlign. For each of the 1361 query read profiles, we selected one read profile from miRRPdb which showed highest alignment score as potential hit for the query read profile. We observed two completely distinct distributions of the alignment scores (Figure 4A), one from alignment of both query and subject read profiles as miRNA (miRNA-miRNA) and another from alignment of any other ncRNA except miRNA as query and miRNA as subject read profile (other-miRNA). ROC curve analysis using R package ROCR (Sing et al., 2005) showed a high AUC of 0.93 suggesting that miRNA read profiles have characteristic features that are distinct from read profiles of other ncRNAs and can be employed for confident prediction of miRNA. (Figure 4B). Indeed, most miRNA-miRNA read profile alignments (223 out of 285) showed an alignment score of $\geq 0.7$ whereas most othermiRNA read profile alignment scores (1049 out of 1076) were $<0.7$. As the False Positive Rate (FPR) tend to increase above the alignment score of 0.7 (yellow-green intersection) in Figure 4B, we chose 0.7 as the default cut-off alignment score to consider an alignment between unannotated with miRNA read profile as significant and unannotated read profile as putative miRNA candidate.

We further estimated the MCC (Matthews et al., 1975), sensitivity and specificity of the method based on the confusion matrix created for 1361 alignments at a cut-off read profile alignment score of 0.7. The MCC is computed using a confusion matrix that is composed of four components (a) 223 miRNAmiRNA read profile alignments with score $\geq 0.7$ as True Positives (TP), (b) 62 miRNA-miRNA read profile alignments with score $<0.7$ as False Negatives (FN), (c) 1049 other-miRNA read profile alignments with score $<0.7$ as True Negatives (TN); and, (d) 27 other-miRNA read profile alignments with score $\geq 0.7$ as False Positives (FP). Based on the confusion matrix, the sensitivity, specificity and MCC of 0.78, 0.97 and 0.80 , respectively was observed. We also performed the 5 -fold cross validation by splitting the benchmark dataset (1361 annotated read profiles) into five equal and evenly distributed (similar ratio of miRNA and non-miRNA read profiles) datasets. For each of the five rounds of cross-validation, we aligned four of the five datasets against miRRPdb and derived the optimal cut-off of alignment score at which maximum MCC is observed. Using the derived cut-off, the performance of the method is evaluated on the remaining fifth dataset. We observed a mean MCC of $0.80 \pm 0.02$ and an AUC of $0.93 \pm 0.02$. A mean cut-off score of 0.7 was observed during the cross-validation and is used hereafter for all the further analysis. In the light of recent reports that many microRNAsized small RNAs are commonly produced not only from miRNA precursors but also from other classes of structured RNAs like snoRNA and tRNA (Kawaji et al., 2008; Taft et al., 2009), the above measures can be regarded as a reasonable estimate of the performance of this approach in the prediction of novel miRNA candidates.

Furthermore, we compared the performance of our method to an already available tool, miRanalyzer that detect miRNAs using short RNA-seq data (Hackenberg et al., 2011). We chose miRanalyzer because it is one of the widely used tool for miRNA prediction using RNA-seq data, has a prediction performance comparable to other miRNA prediction tools such as miRDeep2 (Williamson et al., 2013) and can be readily applied on our benchmark dataset of mapped reads. We evaluated the performance of miRanalyzer on the mapped reads corresponding to 1361 annotated read profiles from our ENCODE dataset using both the default and model mode of miRanalyzer. In default mode, miRanalyzer made predictions by first mapping reads to known miRNAs from miRBase followed by using random forest model for the remaining set of reads. In model mode, all the predictions are exclusively based on random forest model. We

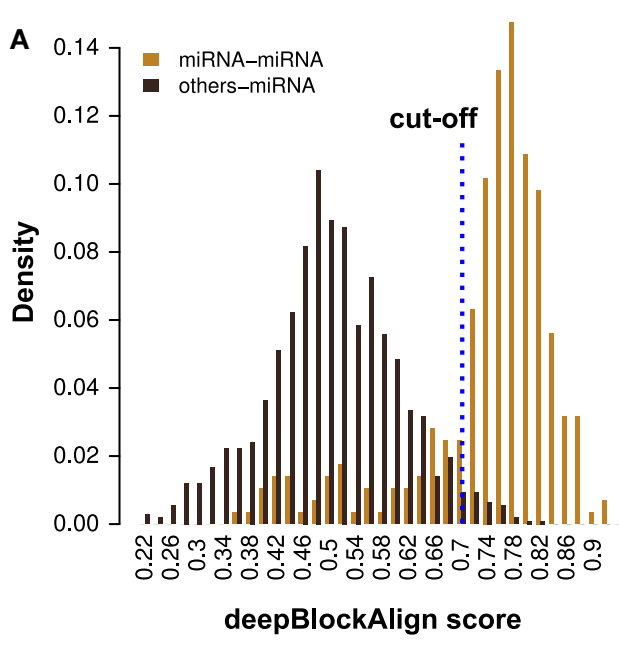

FIGURE 4 | Performance evaluation of the proposed method for the prediction of putative miRNAs using alignment of read profiles. (A) Density distribution of deepBlockAlign alignment scores for 285 miRNA (orange) and 1076 other ncRNA (black) read profiles from ENCODE dataset against miRNA read profile database (miRRPdb), respectively. We observed two distinct score distributions comprised of most miRNAs (223 out of 285)

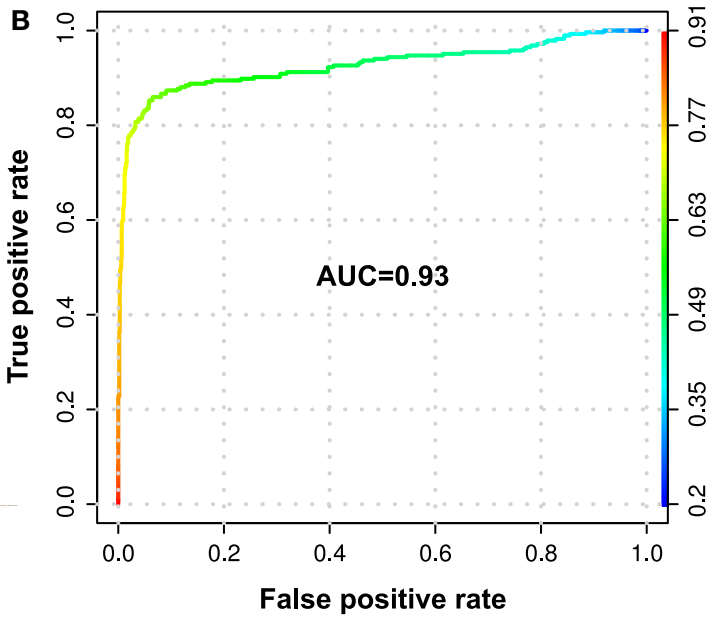

that have an alignment score of $\geq 0.7$ and most of the other ncRNAs (1049 out of 1076) with score $<0.7$ against miRRPdb. (B) ROC curve analysis of the prediction performance. A high AUC of 0.93 was observed suggesting that miRNA read profiles have characteristic features that are distinct from read profiles of other ncRNAs and can be employed for confident miRNA prediction. 
observed an AUC of 0.94 and 0.95 for the former and later mode (Supplementary document available at the web page, http://rth. $\mathrm{dk} /$ resources/mirdba). Out of 223 known miRNAs that were correctly predicted by our method, 160 and 208 were also predicted by miRanalyzer using default and model mode. The high performance using the model mode is not surprising since the models have been trained using random forest on the secondary structure features of the same set of known miRNAs within the benchmark dataset. It is to be noted that while computing the AUC, we considered only those ncRNAs, out of 1361 ncRNAs, that have corresponding reads mapped by the miRanalyzer. When computing the AUC for all the 1361 ncRNAs irrespective of their mapping status, we observed a low AUC of 0.64 and 0.68 for the two modes, respectively.

To further evaluate the performance of our method, we analyzed the short RNA-seq data corresponding to Human HeLa cells (GSE10829) that was used to benchmark the performance of miRanalyzer. We observed an AUC of 0.92 for the dataset which is approximately the same AUC of 0.93 observed for 1361 read profiles from the ENCODE dataset. Considering that our method has not implemented any specific miRNA characteristic feature such as hairpin loop size or length of pre-miRNA, it is intriguing that we obtain a high scoring performance of 0.92 in AUC on the original miRanalyzer dataset. In this context, the performance of miRanalyzer on the HeLa dataset has been reported as an AUC of 0.98. Taken together, we observed comparable performance of our method to that of miRanalyzer suggesting that reasonably confident prediction of miRNAs can be performed based on alignment of read profiles. Furthermore, since many of the miRNAs were predicted exclusively by either of the two methods, the diversity in the two prediction approaches can potentially be applied for an enhanced scheme based on the two methods.

\subsection{IDENTIFICATION OF NEWLY ADDED miRNA ENTRIES IN miRBase}

Since, we used miRBase Release 16 to annotate read profiles from the ENCODE dataset, we checked how many of the newly added miRNAs in miRBase Release 19 are identified by our approach. A total of 558 new human miRNAs has been added in the miRBase since release 16. We observed that out of 558, 49 miRNAs are represented as read profiles in our ENCODE dataset. Of these 49 miRNAs, 35 were observed to a have significant alignment score $(\geq 0.7)$ with miRRPdb, suggesting that the read profile based search can identify most of the newly added miRNAs given that their expression is sufficiently captured during the RNA-seq experiments.

\subsection{PUTATIVE microRNAs}

To predict putative miRNA candidates, we aligned 3434 unannotated read profiles from the ENCODE dataset against miRRPdb using deepBlockAlign. We retrieved 523 alignments with an alignment score of $\geq 0.7$ between an unannotated and miRNA read profile from miRRPdb. Figure 5 shows one such alignment between an unannotated read profile from chr17:2084172020841781(+) and a read profile of hsa-mir-519b. We can observe high similarity between the two read profiles characterized by similar number, size and distance between the read blocks.
Furthermore, the relative arrangement of reads is also very similar between the two, as illustrated through the "Block alignment". We also checked for the consensus RNA secondary structure by extracting the Multiz alignment (Blanchette et al., 2004) corresponding to the unannotated region from the UCSC browser (Figure 5D). The consensus RNA structure is predicted using the PETfold webserver (Seemann et al., 2011), and it showed a highly conserved hairpin loop structure characteristic for miRNA secondary structure. This further suggests that this region potentially can harbor a miRNA. The unannotated status of this region was checked by searching for possible annotations in miRBase, UCSC tracks of ENCODE/GENCODE (Version 7, 10, and 12), RefSeq, tRNA and snoRNAs.

\subsection{PUTATIVE miRNAs ARE LOCATED IN REgIONS THAT HAVE SHORT OR POORLY CONSERVED MAF BLOCKS}

Secondary structure conservation across evolutionary tree is considered a compelling evidence for the biological function of a RNA. Therefore, many tools like Evofold (Pedersen et al., 2006), RNAAlifold (Bernhart et al., 2008), PETfold (Seemann et al., 2008), and RNAz (Gruber et al., 2010) integrate sequence conservation across multiple organisms (Multiz alignments) with RNA folding algorithms for reliable prediction of RNA secondary structure like hairpin loop structure of miRNA. A Multiz alignment represents multiple sequence alignments across a set of species along with measures of evolutionary conservation (Blanchette et al., 2004). For comparative analysis, we retrieved 46-way and 13-way Multiz alignments corresponding to both 523 miRNA candidates and 223 known miRNAs from UCSC (Karolchik et al., 2004) and our in house compilation of Multiz alignments, respectively. The in house 13-way Multiz alignments have been made on 13 representative organisms in the evolutionary tree (Anthon et al., in preparation). As expected, we observed less MAF block fragmentation over the location of 523 miRNA candidates for the 13-way alignment in comparison to the 46-way alignment, see Figure 6.

For the 13-way alignment, we observed that 272 out of the 523 candidates have too short Multiz alignments $(<50 \mathrm{nt})$ in comparison to 49 out of 223 for known miRNAs (Figure 6). Furthermore, Multiz alignments from putative miRNAs were observed to have a significantly lower average pairwise identity in comparison to that from known miRNAs (Figure 6). A similar pattern of either short or a significantly lower average pairwise identity in Multiz alignments from putative miRNA in comparison to known miRNAs was observed for 46-way Multiz alignments. In this context, absence of well-defined Multiz alignments may well contribute to many of these putative miRNAs not being identified through methods based on a set of aligned RNA sequences for the prediction of non-coding RNAs (Gorodkin et al., 2010).

\subsection{SECONDARY STRUCTURE ANALYSIS OF PUTATIVE miRNAs}

To independently analyze the 523 miRNA candidate regions for secondary structure, we processed all the corresponding Multiz blocks for quality such as length and average pairwise identity. Interestingly only 278 Multiz alignments have length $\geq 45 \mathrm{nt}$ and we therefore only carried out prediction on these. Since, 

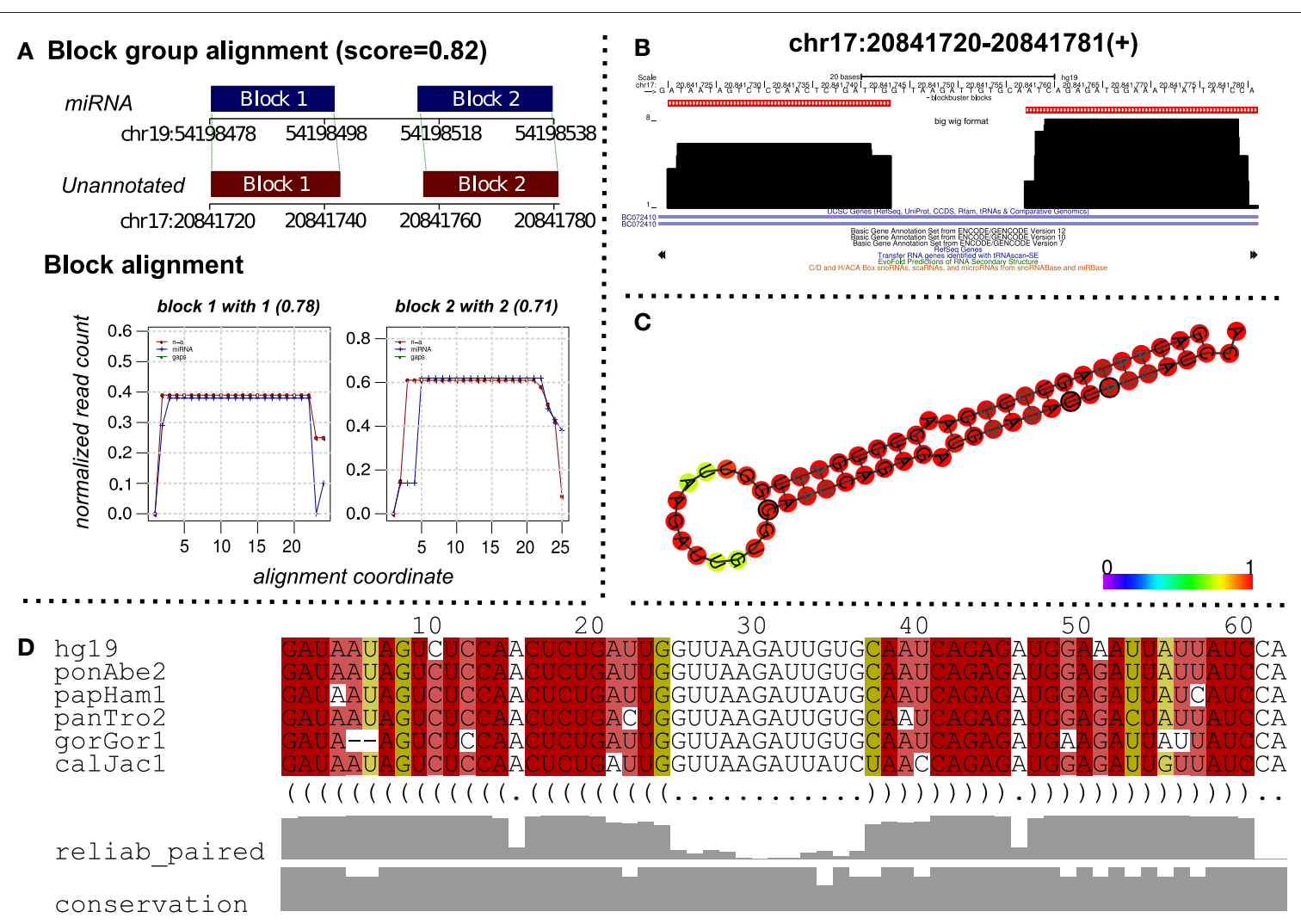

FIGURE 5 | A putative miRNA predicted by alignment of read profiles. (A) Alignment between an unannotated and miRNA read profile (block group) computed using deepBlockAlign. The alignment is obtained while aligning unannotated read profiles from ENCODE dataset against the miRRPdb. The unannotated region has a similar read profile to that of miRNA characterized by similar arrangement of reads as evident from block alignment and similar size and distance between the read blocks, leading to a high alignment score of 0.82 . (B) The read profile for the unannotated region [chr17:20841720-20841781(+)] in UCSC's bigWig format. (C) Consensus RNA secondary structure computed using Multiz alignment with high conservation (Blanchette et al., 2004) from six vertebrate genomes corresponding to the unannotated region. The structure has been predicted using PETfold (Seemann et al., 2011) and has a characteristic hair-pin loop structure of miRNAs. (D) Multiz alignment (Blanchette et al., 2004) across six vertebrate genomes for the predicted consensus RNA secondary structure.
miRNAs usually are $\sim 22 \mathrm{nt}$ in length, a Multiz alignment of length $<45$ can seldom harbor a miRNA hairpin loop structure. Therefore, for the remaining 245 Multiz alignments no attempt to predict RNA secondary structures using sequences from multiple organisms was made. To predict the secondary structure using multiple sequences, we employed two widely used tools, CMfinder (Yao et al., 2006) and RNAz (Gruber et al., 2010), respectively. CMfinder is an expectation maximization algorithm that uses covariance models to predict secondary structure motifs for a set of unaligned sequences (Yao et al., 2006). On the other hand, RNAz is a support vector machine (SVM) based method that evaluates evolutionary conserved pre-aligned set of sequences with thermodynamic stability of RNA secondary structure to detect structural ncRNAs (Gruber et al., 2010). Thus by using these two tools, we aimed to predict secondary structure for regions that are relatively conserved (pre-aligned, RNAz) and regions that are not so well conserved in terms of sequence (unaligned, CMfinder). These methods also complement each other well (Gorodkin et al., 2010).

We used 13-way Multiz alignments corresponding to 278 miRNA candidates as input and considered a $P$-score from
CMfinder $\geq 50$ (FDR, False Discovery Rate of 0.27) (Seemann et al., in Preparation) and a $P$-value from RNAz $>0.9$ ( $z$-score $<-3$ and FDR 0.1) (Gruber et al., 2010) as significant. Based on these thresholds, we predicted conserved RNA secondary structure motifs in 42 putative miRNA candidate regions by CMfinder (17 candidates), RNAz (39 candidates), or by both (14 candidates) CMfinder and RNAz. Out of these 42 putative miRNA candidates, 33 were also predicted as miRNA by miRanalyzer (Hackenberg et al., 2011). Interestingly, for 39 putative miRNA regions predicted to have conserved secondary structure by RNAz, we observed a significantly higher pairwise sequence identity in comparison to rest of the 474 putative miRNA regions (Wilcoxon test, $P$-value $<0.05$ ). This observation again points to the dependency of tools like RNAz on pre-aligned set of sequences for ncRNA prediction as discussed in the previous section. We also observed 13 candidate regions where despite high average pairwise identify of $\geq 90 \%$ in Multiz alignments, no conserved RNA secondary structure was predicted. In conclusion, we obtain 523 novel miRNA candidates of which 42 are further supported by a predicted conserved hairpin loop RNA secondary structure. 

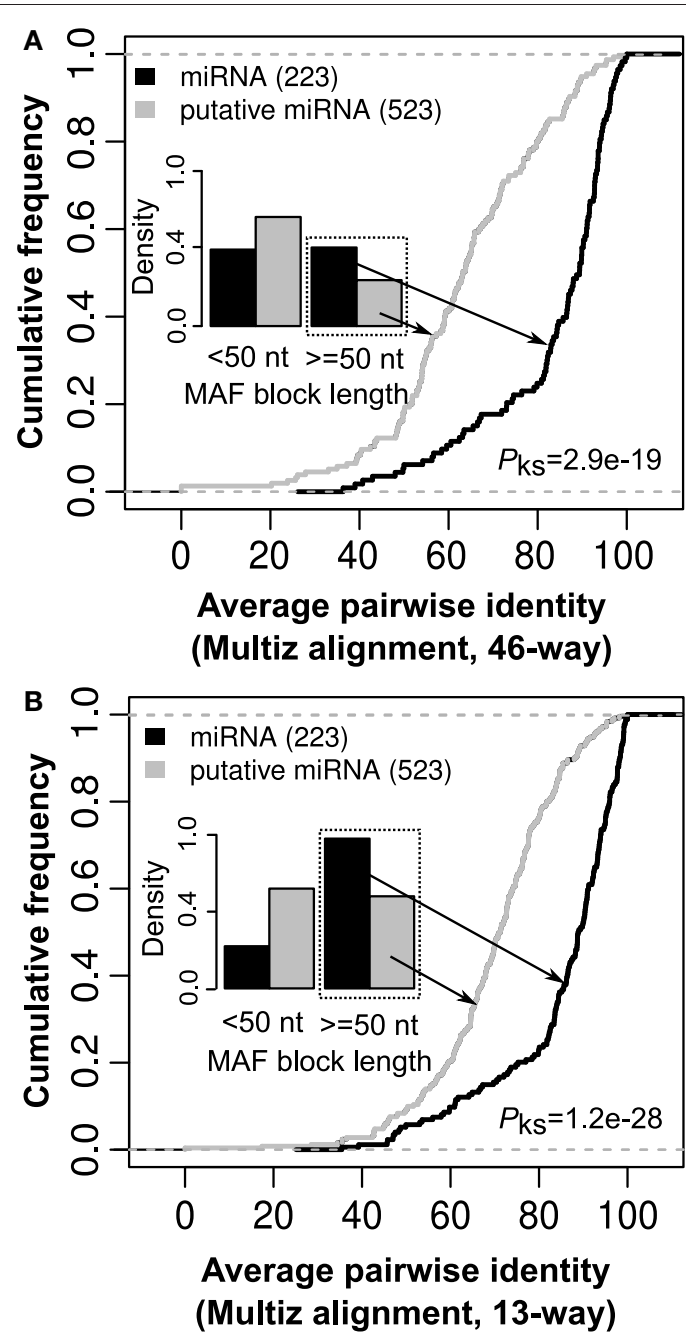

FIGURE 6 | The length and average pairwise identity for Multiz alignments corresponding to known and putative miRNAs. (A) In comparison to known miRNAs, most putative miRNAs have Multiz alignment [46-way downloaded from UCSC (Karolchik et al., 2004)] of length $<50 \mathrm{nt}$ and rest have a significantly low average pairwise identity ( $p$-value $=2.9 \mathrm{e}-19$, Kolmogorov-Smirnov test). (B) For the in house 13-way Multiz alignments (Anthon et al., in preparation), the same pattern was observed with putative miRNAs either have Multiz alignments that are short ( $<50 \mathrm{nt}$ ) or have a significantly lower average pairwise identity in comparison to known miRNAs ( $p$-value $=1.2 \mathrm{e}-28$, Kolmogorov-Smirnov test). This suggests that absence of well-defined Multiz alignments may contribute to many of these putative miRNAs not being identified through methods based on a set of aligned RNA sequences for ncRNA prediction (Gorodkin et al., 2010).

\subsection{TWO DISTINCT CLASSES OF READ PROFILES IN HUMAN AND MOUSE}

On independent clustering of 550 and 624 human and mouse known read profiles from miRRPdb, we observed two wellseparated tree nodes comprised of three constituent clusters of read profiles for both human and mouse, respectively (Figure 7). In mouse, 503 out of $624(81 \%)$ read profiles were represented in the three clusters. In contrast, only 190 out of $550(38 \%)$ read profiles were represented in the three clusters from human. This might well be due to the sequencing depth in RNA-seq experiments from mouse, which is one order of magnitude higher in comparison to human, see Table 3 for details. Higher sequencing depth helps by including only well-defined read blocks while excluding background read noise during preprocessing step (see section 2) thereby leading to well-defined clusters of read profiles.

Nevertheless, in both human and mouse, we observed two classes of read profiles. The first was comprised of characteristic miRNA-like read profiles which had two read blocks with low entropy (Figure 7A) and the second was comprised of read profiles with a significantly high number of read blocks ( $p$-value $<$ 0.05 , Fisher's exact test) and entropy ( $p$-value $<0.05$, Fisher's exact test). Entropy is a measure of the degree of randomness in the arrangement of reads within a read profile (Langenberger et al., 2012, holds the details). An earlier study showed similar findings in Ciona intestinalis where half of the miRNA loci encoded upto four distinct, stable small RNAs. The additional RNAs were shown to be generated from sequences immediately adjacent to the predicted $\sim 60 \mathrm{nt}$ pre-miRNA (Shi et al., 2009). Figure 7B illustrates three example of read profiles that have more than two read blocks, first example has expression in loop region that is interestingly even higher in comparison to that of $\mathrm{miR}^{*}$ and other two examples with expression from region partially overlapping to miR-miR*, a pattern similar to those of miRNA-offset RNAs (moRs) albeit different in not being completely adjacent to the miR-miR* (Shi et al., 2009).

\subsection{READ PROFILES SPECIFIC TO PLANTS AND ANIMALS}

We also performed alignment and hierarchical clustering of all 2540 read profiles in miRRPdb leaving us with 11 distinct clusters of miRNA read profiles (Figure 8). We observed an interesting pattern of read profile distribution across different organisms, while four cluster of read profiles (cluster 361, 371, 335, and 396) were mostly observed in animals ( $h s a, H$. sapiens and $m m u$, M. musculus), three clusters (cluster 389, 386, and 301) were almost exclusively observed in plants (osa, O. sativa and ath, A. thaliana). The rest of the read profiles clusters were observed across both plant and animals. Next, we compared the length and entropy of the read profiles observed specifically in plant and animals. While almost all of the animal specific read profiles were of 60-90 nucleotides in length, plant specific read profiles were either short $(<60 \mathrm{nt})$ or long ( $\geq 90 \mathrm{nt})$ in length. A higher average length of plant pre-miRNA in comparison to human premiRNAs has also been observed in an earlier study (Lindow and Gorodkin, 2007) Plant specific read profiles were also observed to have a significantly higher entropy in comparison to those from animals ( $p$-value $<0.05$, Kolmogorov-Smirnov test). This observation may be attributed to the different biogenesis mechanism of miRNAs in plants and animals (Lelandais-Briere et al., 2010).

\subsection{WEB SERVER FOR MIRNA IDENTIFICATION BASED ON SIMILARITY SEARCH TO miRRPdb}

Based on our proposed method, we developed a web server to predict putative miRNA candidates. The web server is available at http://rth.dk/resources/mirdba and facilitate users to align a query read profile against the database of miRNA read profiles 
(miRRPdb). The standard input is a set of reads mapped to a genomic region of interest in BED format. Users can also adjust several optional parameters that may affect the prediction results, although the default setting is optimal in many cases. The read profile alignment results are presented in a user friendly graphical format and are composed of top two read profiles in miRRPdb that have an alignment score of $\geq 0.7$ (default threshold derived based on benchmarking, see section 3.1) with the query read profile. It should be noted that the alignment score is only an indicator toward similarity of two read profiles. Further checks like similarity in RNA secondary structure should be done to strengthen the prediction results. The alignment of read profiles is visualized using programs developed in PERL, Latex, and R. There is also an option to visualize the read profiles in the UCSC genome browser.

\section{DISCUSSION}

MiRNAs are important regulators of various biological and metabolic processes and computational prediction of miRNA on a genome wide scale is an active research area. We have presented a novel strategy based on alignment of read profiles generated from short RNA-seq data to predict novel miRNA candidates. The alignment of read profiles was performed using a previously published tool, deepBlockAlign. The applicability of the proposed method has been demonstrated by using two short RNA-seq datasets (ENCODE and miRBase datasets). Totals of 4795 and 2540 read profiles were retrieved after preprocessing of ENCODE and miRBase datasets, respectively. 2540 read profiles from miRBase were then compiled to form a database of miRNA read profiles (miRRPdb). Upon alignment of 1361 annotated (285 miRNA and 1024 other ncRNA) read profiles from

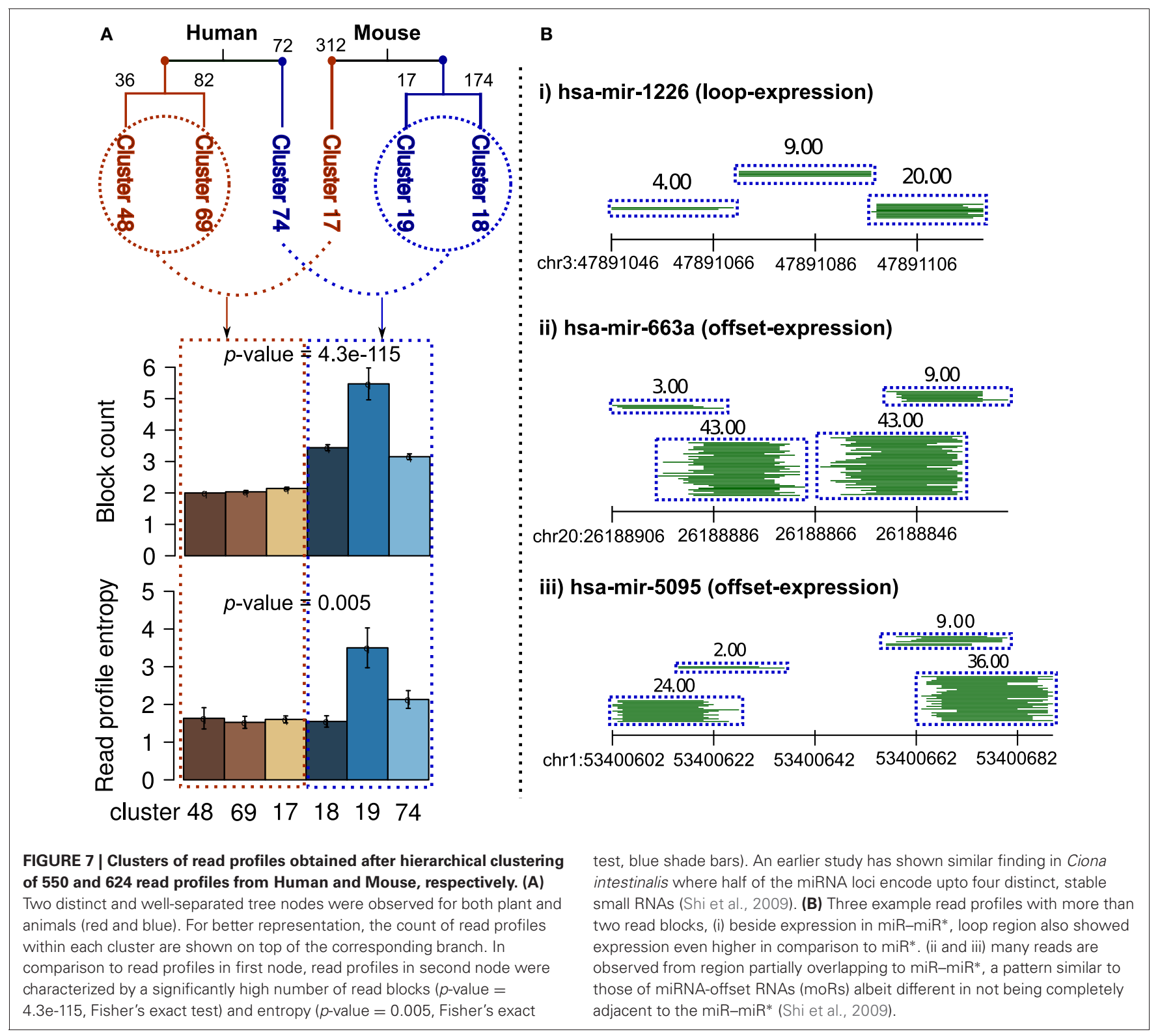




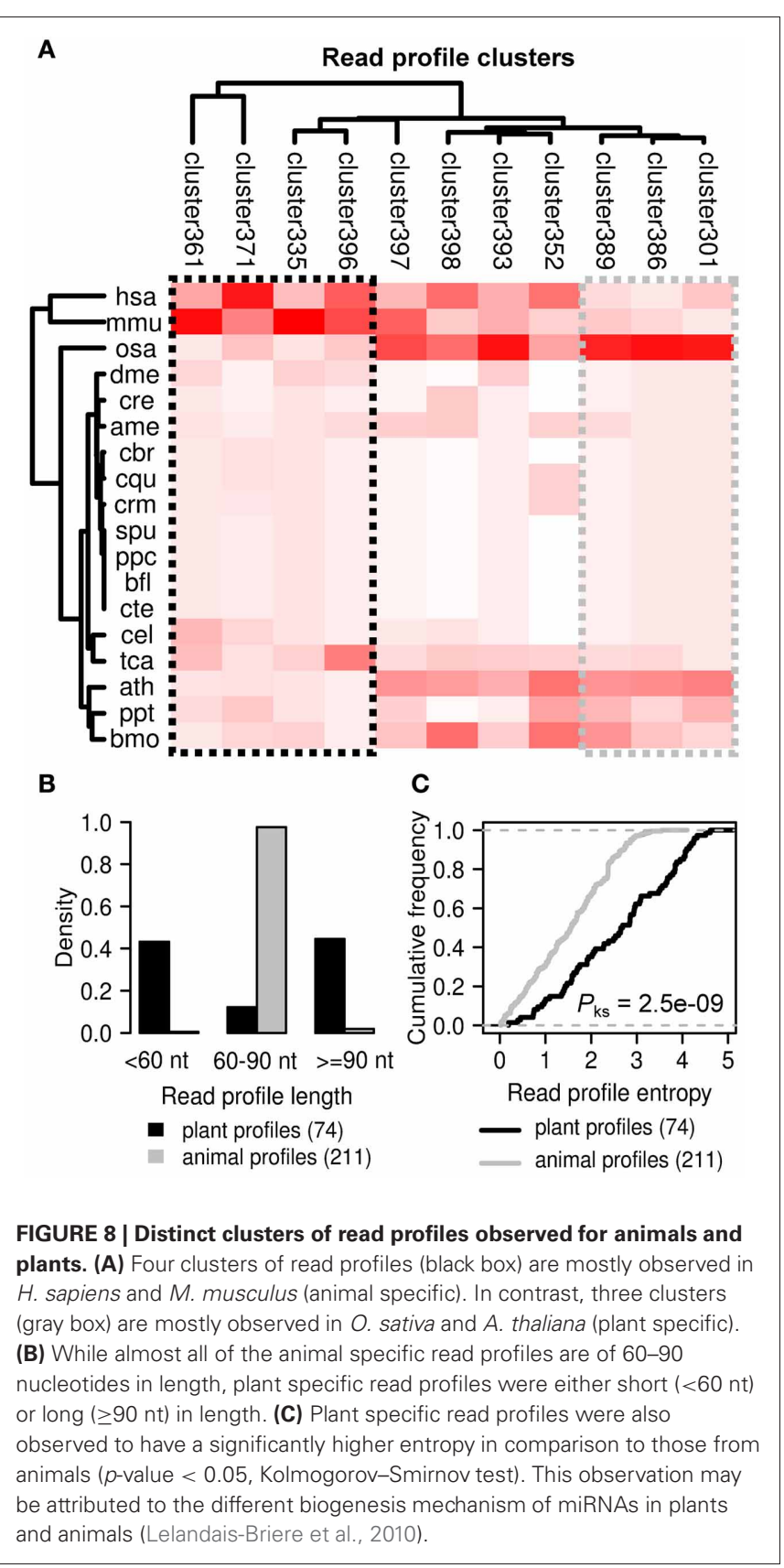

the ENCODE dataset against miRRPdb, we observed two clearly distinct distributions, one comprised of deepBlockAlign scores between non-miRNA against miRNA read profiles and another comprised of scores between miRNA against miRNA read profiles.

Based on this, we computed the deepBlockAlign score that best separates the two distributions, yielding a classification of 0.8 using MCC with sensitivity, specificity and an area under the curve (ROC) of $0.78,0.97$, and 0.93 , respectively. When benchmarking against a representative tool for miRNA prediction based on small RNA-seq data, miRanalyzer (Hackenberg et al., 2011), we observed comparable performance between the two methods on both our dataset as well as the dataset used to benchmark miRanalyzer. Since our method is only based on alignment of read profiles, it has some notable advantages: (a) it can predict putative miRNAs in genomic regions that are devoid of RNA secondary structure information either due to low sequence conservation across multiple organisms that many tools like RNAz (Gruber et al., 2010) require or due to inherent limitation of tools based on single sequence to predict a RNA secondary structure. In this context, many mRNA regions that were predicted to form large, single stranded loops by RNAfold (Gruber et al., 2008) have been shown to form highly base-paired regions using experimental methods (Zheng et al., 2010; Li et al., 2012). (b) it also provides the most similar read profile to a query read profile with respect to arrangement and expression of reads. This can be a useful information, especially when the most similar read profile from miRRPdb is comprised of reads derived from only one experiment, and; (c) similarity search of read profiles can be applied to identify lineage specific miRNA read profiles (see section 3.7) and for the classification of miRNA read profiles based on arrangement of reads (see section 3.6). In total, our method based on alignment of read profiles can be a suitable complement to the other tools such as miRanalyzer and miRDeep that also use short RNA-seq data for miRNA prediction.

Using the proposed method on 3434 uannotated read profiles from ENCODE data set, we predicted 523 unannotated read profiles as putative miRNAs. On RNA secondary structure analysis, 42 of these putative miRNAs were observed to have conserved RNA secondary structure. Furthermore, many of the 523 putative miRNAs were characterized by either short or remarkably low average pairwise identity in corresponding Multiz alignments. Since many RNA secondary structure prediction methods use a set of pre-aligned sequences for ncRNA prediction, absence of well-defined Multiz alignments can contribute to the lack of secondary structure predictions overlapping with many of our miRNA candidates. Global screen of ncRNA candidates using multiple sequence alignment has also been suggested to fail in regions of low sequence similarity (Torarinsson et al., 2006, 2008).

We have shown an unannotated genomic region that share read profile similar to a miRNA along with a characteristic hairpin loop RNA secondary structure with a $3^{\prime}$ overhang. On cluster analysis of read profiles from human and mouse separately, we observed two distinct clusters of read profiles, one with read profiles that have two read blocks and low entropy corresponding to $\mathrm{miR}-\mathrm{miR}^{*}$ and the other with read profiles that have more than two blocks and a significantly higher entropy in comparison to the former. Many of these additional read blocks were observed in regions adjacent or partially overlapping to $\mathrm{miR}-\mathrm{miR}^{*}$ and can be possible candidates for miRNA-offset RNAs (moRs).

On cluster analysis of all 2540 read profiles from 18 different organisms in miRRPdb, we observed 11 distinct clusters of read profiles. Interestingly, four of these read profile clusters were mostly observed in animals and another three read profile clusters were mostly observed in plants. The remaining four read profile clusters were observed in both plant and animals. Furthermore, most of the plant specific read profiles were either too 
short ( $<60 \mathrm{nt})$ or long ( $\geq 90 \mathrm{nt})$ in comparison to animal specific read profiles. Besides, plant read profiles were also observed to have a significantly higher entropy in comparison to the animal read profiles.

Further work includes analysis of miRNA read profiles that have more than two read blocks for potential miRNA-offset miRNAs. In this context, these miRNAs can also be wrongly annotated as miRNA in miRBase. A meta tool integrating the predictions of methods such as miRanalyzer, miRDeep 2 and our method would also be useful for the identification of miRNA candidates that are supported by diverse set of prediction approaches. It would also be of wide interest to classify miRNAs based on read profile patterns that are specific to a given tissue, pathological condition or organism. In this context, closer inspection of plant and animal

\section{REFERENCES}

An, J., Lai, J., Lehman, M., and Nelson, C. (2013). miRDeep*: an integrated application tool for miRNA identification from rna sequencing data. Nucleic Acids Res. 41, 727-737. doi: 10.1093/nar/gks1187

Bartel, D. (2004). MicroRNAs: genomics, biogenesis, mechanism, and function. Cell 116, 281-297. doi: $\quad 10.1016 /$ S0092-8674(04) 00045-5

Bentwich, I. (2005). Prediction and validation of microRNAs and their targets. FEBS Lett. 579, 5904-5910. doi: 10.1016/j.febslet.2005.09.040

Berezikov, E., Guryev, V., van de Belt, J., Wienholds, E., Plasterk, R., and Cuppen, E. (2005). Phylogenetic shadowing and computational identification of human microRNA genes. Cell 120, 21-24. doi: 10.1016/j.cell.2004.12.031

Bernhart, S., Hofacker, I., Will, S., Gruber, A., and Stadler, P. (2008). RNAalifold: improved consensus structure prediction for rna alignments. BMC Bioinform. 9:474. doi: 10.1186/1471-2105-9-474

Blanchette, M., Kent, W., Riemer, C., Elnitski, L., Smit, A., Roskin, K., et al. (2004). Aligning multiple genomic sequences with the threaded blockset aligner. Genome. Res. 14, 708-715. doi: 10.1101/gr.1933104

Chan, P. P., and Lowe, T. M. (2009). GtRNAdb: a database of transfer RNA genes detected in genomic sequence. Nucleic Acids Res. 37, D93-D97. doi: 10.1093/nar/ gkn787

Dezulian, T., Remmert, M., Palatnik, J. F., Weigel, D., and Huson, D. H. (2006). Identification of plant microRNA homologs. Bioinformatics 22, 359-360. doi: 10.1093/bioinformatics/bti802

ENCODE Consortium. (2011). A users guide to the encyclopedia of dna elements (encode). PLoS Biol. 9:e1001046. doi: 10.1371/journal.pbio. 1001046

ENCODE Consortium. (2012). An integrated encyclopedia of DNA elements in the human genome. Nature 489, 57-74. doi: 10.1038/nature11247

Erhard, F., and Zimmer, R. (2010). Classification of ncRNAs using position and size information in deep sequencing data. Bioinformatics 26, i426-i432. doi: 10.1093/bioinformatics/btq363

Findeiß, S., Langenberger, D., Stadler, P. F., and Hoffmann, S. (2011). Traces of post-transcriptional RNA modifications in deep sequencing data. Biol. Chem. 392, 305-313. doi: 10.1515/bc.2011.043

Friedländer, M., Mackowiak, S., Li, N., Chen, W., and Rajewsky, N. (2012). miRDeep2 accurately identifies known and hundreds of novel microRNA genes in seven animal clades. Nucleic Acids Res. 40, 37-52. doi: 10.1093/nar/gkr688

Gardner, P., Daub, J., Tate, J., Moore, B., Osuch, I., Griffiths-Jones, S., et al. (2011). Rfam: Wikipedia, clans and the decimal release. Nucleic Acids Res. 39(Suppl. 1), D141. doi: 10.1093/nar/gkq1129

Gorodkin, J., Hofacker, I. L., Torarinsson, E., Yao, Z., Havgaard, J. H., and Ruzzo, W. L. (2010). De novo prediction of structured RNAs from genomic sequences. Trends Biotech. 28, 9-19. doi: 10.1016/j.tibtech.2009.09.006

Gruber, A., Findeiß, S., Washietl, S., Hofacker, I., and Stadler, P. (2010). "RNAz 2.0: improved noncoding RNA detection," in Pacific Symposium on Biocomputing. (Kamuela, HI: World Scientific), 69-79.

Gruber, A. R., Lorenz, R., Bernhart, S. H., Neuböck, R., and Hofacker, I. L. (2008). The vienna rna

specific read profiles may also reveal novel and organism specific features in their miRNA read profiles.

\section{FUNDING}

This work is funded in part by the Danish Strategic Research Council (Strategic Growth technologies), the Danish Independent Research Council (Technology and Production), and the Danish Center for Scientific Computation.

\section{ACKNOWLEDGMENTS}

We would like to thank the reviewers for their helpful comments and suggestions, Christian Anthon for the help in development of the web server and Betina Wingreen Jensen for proof-reading the manuscript.

websuite. Nucleic Acids Res. 36(Suppl. 2), W70-W74. doi: 10.1093/nar/gkn 188

Hackenberg, M., Rodríguez-Ezpeleta, N., and Aransay, A. M. (2011). miRanalyzer: an update on the detection and analysis of microRNAs in high-throughput sequencing experiments. Nucleic Acids Res. 39(Suppl. 2), W132-W138. doi: 10.1093/nar/ gkr247

Jiang, P., Wu, H., Wang, W., Ma, W., Sun, X., and Lu, Z. (2007). MiPred: classification of real and pseudo microRNA precursors using random forest prediction model with combined features. Nucleic Acids Res. 35(Suppl. 2), W339-W344. doi: 10.1093/nar/gkm368

Jung, C. H., Hansen, M. A., Makunin, I. V., Korbie, D., and Mattick, J. (2010). Identification of novel non-coding RNAs using profiles of short sequence reads from next generation sequencing data. BMC Genomics 11:77. doi: 10.1186/14712164-11-77

Karolchik, D., Hinrichs, A. S., Furey, T. S., Roskin, K. M., Sugnet, C. W., Haussler, D., et al. (2004). The UCSC table browser data retrieval tool. Nucleic Acids Res. 32, D493-D496. doi: 10.1093/nar/gkh103

Kawaji, H., Nakamura, M., Takahashi, Y., Sandelin, A., Katayama, S., Fukuda, S., et al. (2008). Hidden layers of human small RNAs. BMC Genomics 9:157. doi: 10.1186/1471-2164-9-157

Kozomara, A., and Griffiths-Jones, S. (2011). miRBase: integrating microRNA annotation and deep-sequencing data. Nucleic Acids Res. 39, D152-D157. doi: 10.1093/nar/gkq1027

Lai, E. C., Tomancak, P., Williams, R. W., and Rubin, G. M. (2003). Computational identification of drosophila microRNA genes. Genome. Biol. 4:R42. doi: 10.1186/ gb-2003-4-7-r42

Langenberger, D., Bermudez-Santana, C., Hertel, J., Hoffmann, S., Khaitovich, P., and Stadler, P. (2009). Evidence for human microRNA-offset RNAs in small RNA sequencing data. Bioinformatics 25, 2298-2301. doi: 10.1093/bioinformatics/btp419

Langenberger, D., Bermudez-Santana, C. I., Stadler, P. F., and Hoffmann, S. (2010). "Identification and classification of small RNAs in transcriptome sequence data," in Pacific Symposium on Biocomputing (Kamuela, HI), 80-87.

Langenberger, D., Pundhir, S., Ekstrøm, C., Stadler, P., Hoffmann, S., and Gorodkin, J. (2012). deepblockalign: a tool for aligning rnaseq profiles of read block patterns. Bioinformatics 28, 17-24. doi: 10.1093/bioinformatics/btr598

Lelandais-Briere, C., Sorin, C., Declerck, M., Benslimane, A., Crespi, M., and Hartmann, C. (2010). Small RNA diversity in plants and its impact in development. Curr. Genom. 11, 14. doi: 10.2174/138920210790217918

Li, F., Zheng, Q., Ryvkin, P., Dragomir, I., Desai, Y., Aiyer, S., et al. (2012). Global analysis of RNA secondary structure in two metazoans. Cell Rep. 1, 69-82. doi: 10.1016/j.celrep.2011.10.002

Lim, L. P., Lau, N. C., Weinstein, E. G., Abdelhakim, A., Yekta, S., Rhoades, M. W., et al. (2003). The microRNAs of caenorhabditis elegans. Genes Dev. 17, 991-1008. doi: 10.1101/gad.1074403

Lindow, M., and Gorodkin, J. (2007). Principles and limitations of computational microRNA gene and target finding. DNA Cell Biol. 26, 339-351. doi: 10.1089/dna.2006.0551 
Matthews, B. (1975). Comparison of the predicted and observed secondary structure of $\mathrm{t} 4 \mathrm{\text {phage }}$ lysozyme. Biochim. Biophys. Acta 405, 442. doi: 10.1016/0005-2795 (75)90109-9

Nam, J.-W., Shin, K.-R., Han, J., Lee, Y., Kim, V. N., and Zhang, B.-T. (2005). Human microRNA prediction through a probabilistic colearning model of sequence and structure. Nucleic Acids Res. 33, 3570-3581. doi: 10.1093/nar/gki668

Ohler, U., Yekta, S., Lim, L. P., Bartel, D. P., and Burge, C. B. (2004). Patterns of flanking sequence conservation and a characteristic upstream motif for microRNA gene identification. RNA 10, 1309-1322. doi: 10.1261/rna.5206304

Pedersen, J., Bejerano, G., Siepel, A., Rosenbloom, K., LindbladToh, K., Lander, E., et al. (2006). Identification and classification of conserved RNA secondary structures in the human genome. PLoS Comput. Biol. 2:e33. doi: 10.1371/journal.pcbi.0020033

Seemann, S., Gorodkin, J., and Backofen, R. (2008). Unifying evolutionary and thermodynamic information for RNA folding of multiple alignments. Nucleic Acids Res. 36, 6355-6362. doi: 10.1093/nar/gkn544

Seemann, S., Menzel, P., Backofen, R., and Gorodkin, J. (2011). The PETfold and PETcofold web servers for intra-and intermolecular structures of multiple rna sequences. Nucleic Acids Res. 39(Suppl 2), W107-W111. doi: 10.1093/nar/gkr248

Sheng, Y., Engström, P. G., and Lenhard, B. (2007). Mammalian microRNA prediction through a support vector machine model of sequence and structure. PLoS ONE 2:e946. doi: 10.1371/journal.pone.0000946

Shi, W., Hendrix, D., Levine, M. and Haley, B. (2009). A distinct class of small RNAs arises from pre-miRNA-proximal regions in a simple chordate. Nat. Struct. Mol. Biol. 16, 183-189. doi: 10.1038/nsmb.1536

Sing, T., Sander, O., Beerenwinkel, N., and Lengauer, T. (2005). Rocr: visualizing classifier performance in $\mathrm{r}$. Bioinformatics 21, 3940-3941. doi: 10.1093/bioinformatics/bti623

Suzuki, R., and Shimodaira, H. (2006). Pvclust: an $\mathrm{R}$ package for assessing the uncertainty in hierarchical clustering. Bioinformatics 22, 1540-1542. doi: 10.1093/bioinformatics/btl117

Taft, R. J., Glazov, E. A., Lassmann, T., Hayashizaki, Y., Carninci, P., and Mattick, J. S. (2009). Small RNAs derived from snoRNAs. RNA 15, 1233-1240. doi: 10.1261/rna.1528909

Torarinsson, E., Sawera, M., Havgaard, J. H., Fredholm, M., and Gorodkin, J. (2006). Thousands of corresponding human and mouse genomic regions unalignable in primary sequence contain common
RNA structure. Genome. Res. 16, 885-889. doi: 10.1101/gr.5226606

Torarinsson, E., Yao, Z., Wiklund, E., Bramsen, J., Hansen, C., Kjems, J., et al. (2008). Comparative genomics beyond sequence-based alignments: RNA structures in the ENCODE regions. Genome. Res. 18, 242-251. doi: 10.1101/gr.6887408

Wang, X., Zhang, J., Li, F., Gu, J., He, T., Zhang, X., et al. (2005). MicroRNA identification based on sequence and structure alignment. Bioinformatics 21, 3610-3614. doi: 10.1093/bioinformatics/bti562

Williamson, V., Kim, A., Xie, B., McMichael, G. O., Gao, Y., and Vladimirov, V. (2013). Detecting miRNAs in deep-sequencing data: a software performance comparison and evaluation. Brief. Bioinform. 14, 36-45. doi: 10.1093/bib/ bbs010

Yao, Z., Weinberg, Z., and Ruzzo, W. L. (2006). CMfinder- a covariance model based RNA motif finding algorithm. Bioinformatics 22, 445-452. doi: 10.1093/bioinformatics/btk008

Zhang, B., Pan, X., Cox, S., Cobb, G., and Anderson, T. (2006a). Evidence that miRNAs are different from other RNAs. Cell. Mol. Life Sci. 63, 246-254. doi: 10.1007/s00018-0055467-7

Zhang, B., Pan, X., Wang, Q., Cobb, G., and Anderson, T. (2006b). Computational identification of microRNAs and their targets. Comput. Biol. Chem. 30, 395-407. doi: 10.1016/j.compbiolchem.2006. 08.006

Zheng, Q., Ryvkin, P., Li, F., Dragomir, I., Valladares, O., Yang, J., et al. (2010). Genome-wide doublestranded rna sequencing reveals the functional significance of base-paired rnas in arabidopsis. PLoS Genet. 6:e1001141. doi: 10.1371/journal.pgen.1001141

Conflict of Interest Statement: The authors declare that the research was conducted in the absence of any commercial or financial relationships that could be construed as a potential conflict of interest.

Received: 01 February 2013; paper pending published: 26 March 2013; accepted: 21 June 2013; published online: 11 July 2013.

Citation: Pundhir $S$ and Gorodkin J (2013) MicroRNA discovery by similarity search to a database of RNA-seq profiles. Front. Genet. 4:133. doi: 10.3389/ fgene.2013.00133

This article was submitted to Frontiers in Bioinformatics and Computational Biology, a specialty of Frontiers in Genetics.

Copyright (C) 2013 Pundhir and Gorodkin. This is an open-access article distributed under the terms of the Creative Commons Attribution License, which permits use, distribution and reproduction in other forums, provided the original authors and source are credited and subject to any copyright notices concerning any third-party graphics etc. 